\title{
National survey of blindness and low vision in The Gambia: Results
}

\author{
H FAAL, ${ }^{1}$ D MINASSIAN, ${ }^{2}$ S SOWA ${ }^{3}$ AND A FOSTER ${ }^{2}$ \\ From the ${ }^{1}$ Royal Victoria Hospital, Banjul; the ${ }^{2}$ International Centre for Eye Health, Institute of Ophthalmology, \\ London; and ${ }^{3}$ Pieds Nus, Caubon St Sauveur, Duras 47120, France
}

SUMMARY A population based survey of blindness and eye disease has been conducted throughout the whole country of The Gambia, and 8174 people were examined. The prevalence of blindness (best acuity less than $3 / 60$ ) was $0.7 \%$ and low vision $(6 / 24-3 / 60) 1.4 \%$. The causes of blindness were cataract $(55 \%)$, non-trachomatous corneal opacity/phthisis $(20 \%)$, and trachoma (17\%). An estimated 5500 people in The Gambia require cataract surgery, 4600 eyelid surgery for entropion, and 4600 people spectacles to correct a refractive error which causes a visual acuity of less than 6/18. More than half the current burden of blindness in The Gambia is potentially remediable through the provision of cataract surgery and aphakic spectacles.

The Gambia is a small country of approximately 800000 people situated on the west coast of Africa. A population based survey of blindness and eye disease has been conducted throughout the whole country, initiated by the Ministry of Health in The Gambia, in collaboration with the International Centre for Eye Health, London, and the Prevention of Blindness Programme of the World Health Organisation.

The survey was undertaken as part of the first phase in the development of a National Eye Care Programme in order to establish baseline data on the prevalence of the major blinding diseases. This information will assist in the planning and future evaluation of eye services and blindness control programmes.

\section{Material and methods}

The Gambia is divided into three administrative health regions-Western, Central, and Eastern. A population based prevalence survey of blindness and eye disease was conducted during March-May 1986 on a countrywide sample of approximately $1 \%$ of the total population (Fig. 1).

A multistage cluster random sampling procedure was used with stratification by (1) district, subgrouping north and south of the river; (2) urban, periurban,

Correspondence to Dr A Foster, International Centre for Eye Health, Institute of Ophthalmology, 27-29 Cayton Street, London EC1V 9EJ. and rural communities; and (3) settlement size (under 300 and 300 or more).

The only exclusion area was part of the periurban area of the capital, Banjul, which is inhabited by foreigners with high incomes. A poor slum area, and a typical periurban area of the capital were surveyed. The 1983 census figures for The Gambia provided the sampling frame for the study. Fifty two communities were randomly chosen to reach the required sample size of at least 8000 . The sample provided 8696 persons, of whom 8174 were examined.

Data were collected by means of the Eye Examination Record (Version II) developed by the World Health Organisation Prevention of Blindness Programme. This form was evaluated by the team as part of the study. Two teams worked side by side, with each team consisting of an ophthalmologist, ophthalmic assistant, one general nurse, two eye auxiliaries, two enumerators, and a driver. Demographic data were recorded by the enumerators, visual acuities measured by the eye auxiliaries, and ocular examination, including assessment for trachoma (all ages) and xerophthalmia (0-6 years), performed by the ophthalmic assistants and ophthalmologists. Any individual with a visual acuity of less than $6 / 18$ in either eye was examined by the ophthalmologist, and the cause of low vision $(6 / 24-3 / 60)$ or blindness (less than 3/60) was recorded. Any action required (medical treatment, surgery, or spectacles) as identified by the examination team was also recorded. Further details on the project outline, design and 


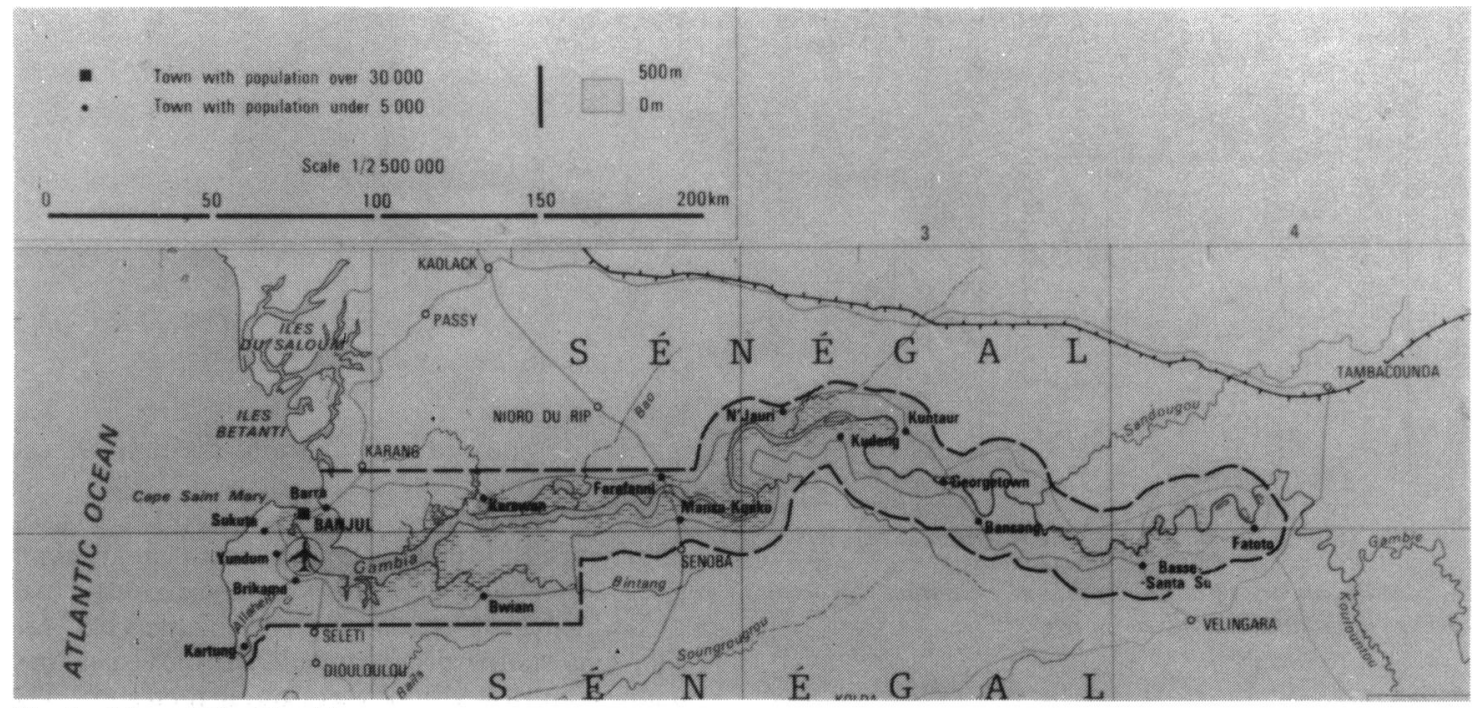

Fig. 1 Map of The Gambia.

methodology of the survey, and evaluation of the eye examination record are given elsewhere. ${ }^{12}$

\section{Results}

Table 1 gives data on the census population and coverage of the required sample, which ranged from $86 \%$ in the capital to $97 \%$ in the Eastern region, with an overall coverage of $94 \%$.

The data estimated the prevalence of blindness defined as a binocular visual acuity of less than $3 / 60$ to be $0.7 \%$ (5500 people in The Gambia) and prevalence of low vision (best acuity $6 / 24-3 / 60$ ) to be $1.4 \%$ (11000 people in The Gambia). The total number of blind and visually impaired was 16500 (approximate 95\% confidence limits 14000-19000). The prevalence of blindness by age in The Gambia is given in Fig. 2, but the figures do not account for blind children who might have been staying in blind schools.

The periurban area of Banjul shows much less visual impairment and blindness than the poorer

Table 1 National survey of blindness and eye disease, The Gambia 1986

\begin{tabular}{lccc}
\hline Region & Population & $\begin{array}{l}\text { Number } \\
\text { examined }\end{array}$ & Coverage \\
\hline Eastern & 282000 & 2606 & $97 \%$ \\
Central & 136000 & 2812 & $96 \%$ \\
Western & & & \\
$\quad$ Banjul & 50000 & 412 & $86 \%$ \\
$\quad$ Districts & 332000 & 3140 & $90 \%$ \\
Total & 800000 & 8174 & $94 \%$ \\
\hline
\end{tabular}

slum area of the capital. The prevalence of blindness and low vision in the rural areas is similar to that in the poor urban area of Banjul (Fig. 3).

Unoperated cataract was responsible for $45 \%$ of the blindness in The Gambia and 57\% of low vision. Uncorrected aphakia was the cause of $8 \%$ of blindness and $10 \%$ of low vision. Two per cent of the blindness was due to irreversible blindness from neglected cataract or from the complications of traditional cataract surgery (couching), so that a total of $55 \%$ of blindness (Fig. 4) and $67 \%$ of low vision (Fig. 5) could be attributed to cataract and its complications.

The prevalence of blindness from cataract was minimal in Banjul and in its periurban population. In the sample from these areas no blind person was found that required cataract surgery or aphakic correction, suggesting that these communities were well served by present services being offered by the Eye Department at the Royal Victoria Hospital, Banjul.

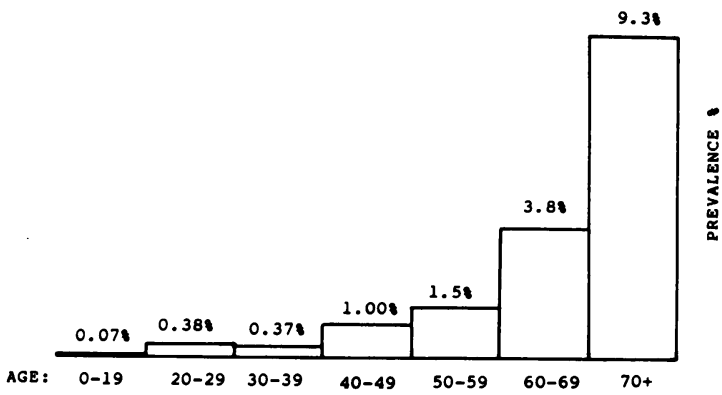

Fig. 2 Prevalence of blindness by age: The Gambia; 1986. 


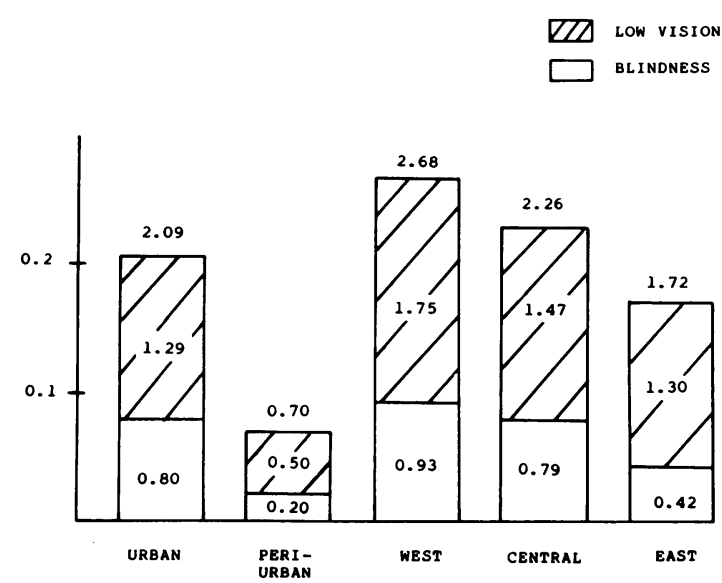

Fig. 3 Prevalence of blindness and low vision in five areas of The Gambia, standardised for age and sex.

Trachoma was responsible for $17 \%$ of the blindness (Fig. 4). The risk of blindness and low vision from trachoma was more than three times higher in females than males (Table 2). The prevalence of trichiasis and entropion varied from $0.6 \%$ in the periurban area of Banjul to $1.8 \%$ in the rural Western Health District.

Non-trachomatous corneal opacity and bilateral phthisis bulbi were responsible for $20 \%$ of the blindness (Fig. 4). (This group excludes people with trachomatous corneal opacity or phthisis due to trauma.) It was not possible certainly to attribute any of the corneal blindness to vitamin A deficiency, though this was a possibility. The prevalence of Bitot spot formation (XIB) in children under 6 years of age was approximately two per 1000 .

The survey data estimated $2 \%$ of blindness and $1 \%$ of low vision in The Gambia to be due to glaucoma. The prevalence of chronic glaucoma was $0.2 \%$ in people over 40 years of age. However, the true prevalence of glaucoma was probably much higher, as visual assessment in this survey was based only on

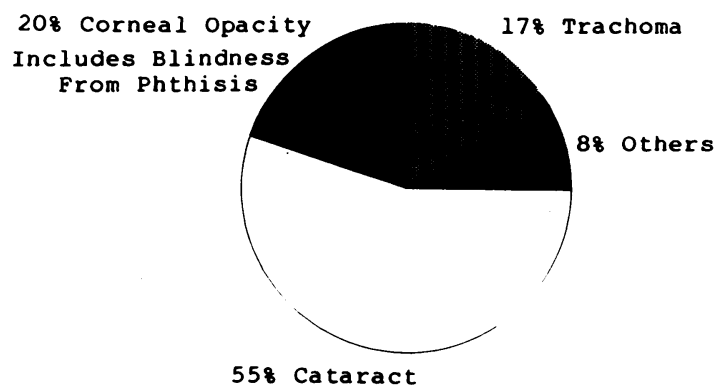

Fig. 4 Causes of blindness in The Gambia, 1986.

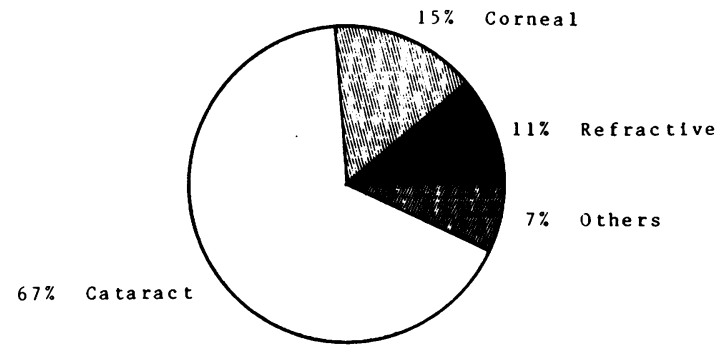

Fig. 5 Causes of low vision in The Gambia, 1986.

visual acuity and did not take into account assessment of visual field.

$6 \%$ of the blindness was due to other causes including optic atrophy, retinal diseases, and trauma (Fig. 4). 11\% of low vision was due to refractive errors which were potentially remediable (Fig. 5).

The age standardised prevalence of visual loss (blindness and low vision) was $1.6 \%$ in men and $2.5 \%$ in women (M:F 1:1.6) (Table 2). The excess visual loss in females could be attributed mainly to cataract and trachoma. The data supported the evidence that males were more likely to seek surgical treatment and aphakic correction for their cataract than females.

Cataract was responsible for nearly half the cases of unilateral blindness and corneal scar for $16 \%$ (Fig. 6 ). $5 \%$ of the population ( 40,000 people) were blind in one eye. The prevalence of uniocular blindness in children (0-14 yr) was $0.3 \%$ (estimated 1200 children in The Gambia). Corneal opacity and phthisis bulbi accounted for $70 \%$ of children with uniocular blindness (Fig. 7).

The eye examination record included information on what kind of treatment, if any, was required by the population sample. These data allowed an estimate to be made of essential ophthalmic services in The Gambia (Table 3).

\section{Discussion}

The $94 \%$ overall coverage rate of a randomised cluster sample allows reliable estimates for the prevalence and causes of blindness and low vision in

Table 2 Age standardised prevalence of visual loss by cause

\begin{tabular}{|c|c|c|c|c|}
\hline & \multirow{2}{*}{$\begin{array}{l}\text { Prevalence } \\
\text { in females }\end{array}$} & \multirow{2}{*}{$\begin{array}{l}\text { Prevalence } \\
\text { in males }\end{array}$} & \multicolumn{2}{|c|}{ Prevalence } \\
\hline & & & Ratio & Difference \\
\hline Trachoma & $0 \cdot 20 \%$ & $0.057 \%$ & $3 \cdot 51$ & $0 \cdot 14 \%$ \\
\hline Cataract & $0.94 \%$ & $0.690 \%$ & $1 \cdot 36$ & $0.32 \%$ \\
\hline $\begin{array}{l}\text { Uncorrected } \\
\text { aphakia }\end{array}$ & $0.15 \%$ & $0.086 \%$ & $1 \cdot 74$ & $0.064 \%$ \\
\hline Other corneal & $0 \cdot 10 \%$ & $0.057 \%$ & $1 \cdot 75$ & $0.043 \%$ \\
\hline
\end{tabular}




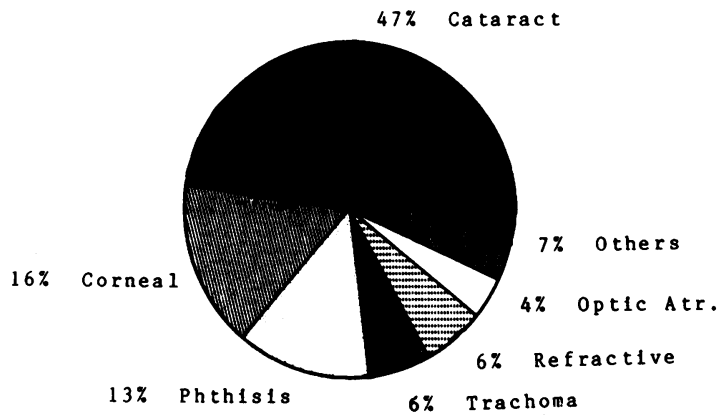

Fig. 6 Causes of uniocular blindness in The Gambia, 1986.

The Gambia. This is the first such national survey undertaken in Africa. The results are consistent with similar surveys undertaken in parts of Malawi ${ }^{3}$ and Kenya. ${ }^{4}$

The Gambia with a population of just under a million and one ophthalmologist provides a suitable model for many countries in sub-Saharan Africa, in which there is on average one ophthalmologist per million population. ${ }^{6}$

The Gambia does not have the special ophthalmological problems found in some areas of West Africa where onchocerciasis is endemic and responsible for a large proportion of blindness. Trachoma, although responsible for $17 \%$ of blindness in The Gambia, is not the hyperendemic disease that is seen in many arid parts of Africa. The data from The Gambia therefore demonstrate the pattern of eye problems that are seen in African countries which have limited health services and ophthalmological care.

\section{CATARACT}

Cataract is the single most important cause of blindness and low vision. An estimated 5500 patients require cataract surgery in The Gambia, but the annual cataract extraction rate is less than 300

Table 3 Summary of the need for basic ophthalmic services in The Gambia

Estimated number of people in need of treatment

$\begin{array}{lc}\text { Cataract surgery } & 5500 \\ \text { Eyelid surgery } & 4600 \\ \text { Need spectacles (distances) } & 4600^{*} \\ \text { Medical eye treatment } & 83000 \\ \text { Other surgical or medical treatment } & 6500\end{array}$

*Does not include presbyopes who may need spectacles for close work, or the estimated 5500 people who will need glasses after cataract surgery.

These numbers represent the current backlog of people in need of the basic eye care.

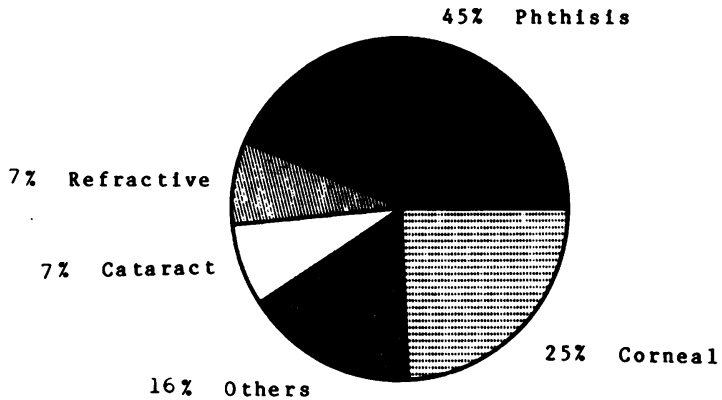

Fig. 7 Causes of uniocular blindness in children (under 15 years) in The Gambia, 1986.

operations per year. At present there is one full-time ophthalmologist in The Gambia with occasional support from visiting Chinese or Western ophthalmologists. Dealing with the problem of cataract blindness will require three to four permanent 'cataract surgeons.' The possible alternatives are to train Gambian ophthalmologists, to teach medical practitioners how to perform cataract surgery, or to train ophthalmic assistants as cataract surgeons. One ophthalmic assistant has already been trained in cataract surgery, and a Gambian doctor is now to start training as an ophthalmologist.

Uncorrected aphakia is responsible for $8 \%$ of blindness and $10 \%$ of low vision. It is essential that, as blind people with cataract receive their operation, facilities are also available to provide at least basic spherical aphakic spectacles. At present there is one optical workshop in the capital, Banjul, producing spherical aphakic spectacles. It is planned that this workshop will be expanded to deal with the increased need. As more ophthalmic assistants are trained and posted to the district hospitals in rural areas, they will be provided with aphakic spectacles from the optical workshop in Banjul for distribution to post-cataract patients.

\section{TRACHOMA}

Trachoma is responsible for $17 \%$ of blindness. The prevalence of visual loss from trachoma in women was $0.20 \%$, which is 3.5 times higher than that in men of the same age. An estimated 4600 patients require surgery for entropion. There are no accurate figures on how many operations are being done each year at present, but it is almost certainly well below 500 a year. The training of eye nurses and ophthalmic assistants in one appropriate operation for upper eyelid entropion could do much to reduce the prevalence of visual loss from trachoma in the future. Two ophthalmic assistants have already been trained to perform entropion surgery, and it is planned to train more in the future. 
NON - TRACHOMATOUS CORNEAL SCAR

On the assumption that bilateral phthisis bulbi is a result of bilateral corneal ulceration and not bilateral trauma, then non-trachomatous corneal scar/phthisis was responsible for $20 \%$ of blindness in adults. Seventy per cent of uniocular blindness in children in the survey was due to corneal scar or phthisis. There were too few children blind in both eyes to give data on the causes of bilateral blindness, but a subsequent examination of 14 children at the blind school in Banjul showed four cases to be due to bilateral corneal scarring. It is not possible from the data to determine accurately the aetiological factors leading to corneal ulceration and blindness in these two groups, but measles, vitamin A deficiency, the use of harmful traditional eye medicines, and ophthalmia neonatorum are the most likely causes of bilateral ulcers; and trauma, herpes simplex, and bacterial and fungal infection are likely to be responsible for the majority of the uniocular corneal scars.

The reduction in blindness from corneal ulceration requires action at the level of maternal and child health services to increase measles vaccination, improve child nutrition, and avoid the use of harmful traditional medicines by ensuring that safe eye medicines such as tetracycline eye ointment are readily available to the population.

Medical workers need to be trained in the diagnosis and urgent management of corneal ulceration in children and adults. The training of community health nurses in activities to prevent trachoma and corneal blindness started in May 1987 and is planned to continue. The training includes the diagnosis and treatment of acute red eye, as well as promotive and preventive measures to improve eye health. Further work is required on the relevant importance of trauma and traditional eye medicines in causing bacterial and fungal suppurative keratitis.

\section{OTHER CAUSES}

Glaucoma was responsible for only $2 \%$ of the blindness and $1 \%$ of low vision. However, this is undoubtedly an underestimate, as the survey examination assessed visual loss only in terms of reduced visual acuity without systematic measurement of visual field in the sample population. But this figure is much less than the findings from previous studies in Nigeria $20 \%$, Kenya $12 \%$, and Tanzania $14 \% .{ }^{467}$ The reasons for these differences are not known, though genetic factors may be important.

A substantial proportion of low vision in The Gambia $(11 \%)$ is due to uncorrected refractive errors. This cause of visual loss is potentially remediable by the provision of appropriate optical services. An estimated 4600 people need spectacles for distance correction because their visual acuity is less than $6 / 18$. The majority of these people $(90 \%)$ live in rural communities. This does not include people requiring aphakic or presbyopic corrections.

Other causes of blindness and low vision include trauma, which was responsible for $2 \%$ of blindness; and optic atrophy, which was responsible for $2 \%$ of blindness and $1 \%$ of low vision.

\section{Conclusion}

The Gambia, with a population of 800000 and one ophthalmologist, provides a model for many parts of Africa, enabling us to identify the important ophthalmic problems and develop relevant eye services to reduce the number of needlessly blind people.

Cataract is the major cause of blindness (estimated 7000 people/million population requiring cataract surgery). To meet this urgent need a minimum of three to four cataract surgeons per million population is initially required in African countries.

Corneal scarring from trachoma and corneal ulceration is the second cause of blindness. These conditions are largely preventable through immunisation programmes, nutrition education, improved personal hygiene, better community sanitation, and the provision of tetracycline eye ointment and vitamin A capsules at the primary health care level.

More than half $(53 \%)$ of the current burden of blindness in The Gambia is potentially remediable through the provision of cataract surgery and aphakic spectacles. 5500 people in The Gambia (7000/million population) require cataract operation.

The challenge is to develop basic curative and preventive ophthalmic services that are accessible to, and will be taken up by, the majority of the rural population. These services are now being developed and extended in The Gambia as part of the National Eye Care Programme.

This work was supported by the World Health Organisation's programme for the Prevention of Blindness under contract no. No1-EY-9-2103 with the National Institutes of Health/National Eye Institute, Bethesda, USA.

\section{References}

$1 \mathrm{Faal} \mathrm{H}$, Minassian D. National survey of blindness and eye disease in The Gambia 1986: Design and methodology. London: International Centre for Eye Health, 1986.

2 Faal H, Minassian D, Foster A. Evaluations of the WHO protocol for surveying eye disease and blindness. London: International Centre for Eye Health, 1986.

3 Chirambo MC, Tielsch JM, West KP, et al. Blindness and visual impairment in Southern Malawi. Bull WHO 1986; 64: 567-72.

4 Whitfield R, et al. Experiences of the Kenya Rural Blindness Prevention Project. Washington, DC: International Eye Foundation, 1981.

5 Minassian DC, Schemann JF. Prevalence and causes of blindness in Togo. Geneva: Report to the WHO Prevention of Blindness Programme, 1986. 
6 Foster A. Cataract Blindness in Africa. Ophthalmic Surg 1987; 18: 384-8.

7 Olurin O. Primary glaucoma in Nigeria. East Afr Med J 1972; 49: 725-34. 